\title{
Erratum to: Cardiac dysfunction induced by weaning from mechanical ventilation: incidence, risk factors, and effects of fluid removal
}

Jinglun Liu' ${ }^{1,2,3,4 \dagger}$, Feng Shen ${ }^{1,2,3,5 \dagger}$, Jean-Louis Teboul ${ }^{1,2,3}$, Nadia Anguel ${ }^{1,2,3}$, Alexandra Beurton ${ }^{1,2,3}$, Nadia Bezaz ${ }^{1,2,3}$, Christian Richard ${ }^{1,2,3}$ and Xavier Monnet ${ }^{1,2,3^{*}}$

\section{Erratum}

Unfortunately this article was published with an error. During production of the original article [1], "with WiPO" and "without WiPO" on " $\triangle \mathrm{CI} \%$ during PLR" has been switched on Fig. 3. The correct figure is shown below.

\section{Author details}

${ }^{1}$ Université Paris-Sud, Faculté de Médecine, Université Paris-Saclay, Le Kremlin-Bicêtre, France. ${ }^{2}$ AP-HP, Service de réanimation médicale, Hôpital de Bicêtre, 78, rue du Général Leclerc, 94270 Le Kremlin-Bicêtre, France. ${ }^{3}$ Inserm UMR_S 999, Hôpital Marie Lannelongue, Le Plessis-Robinson, France.

${ }^{4}$ Department of Emergency Medicine and Critical Care Medicine, The First Affiliated Hospital of Chongqing Medical University, Chongqing, China.

${ }^{5}$ Department of Critical Care Medicine, Affiliated Hospital of Guizhou Medical University, Guiyang, China.

Published online: 08 March 2017

\section{Reference}

1. Monnet X. Cardiac dysfunction induced by weaning from mechanical

ventilation: incidence, risk factors, and effects of fluid removal. BMC Critical Care. 2016:20:369. doi:10.1186/s13054-016-1533-9.

\footnotetext{
* Correspondence: xavier.monnet@aphp.fr

${ }^{\dagger}$ Equal contributors

${ }^{1}$ Université Paris-Sud, Faculté de Médecine, Université Paris-Saclay, Le Kremlin-Bicêtre, France

${ }^{2}$ AP-HP, Service de réanimation médicale, Hôpital de Bicêtre, 78, rue du

Général Leclerc, 94270 Le Kremlin-Bicêtre, France

Full list of author information is available at the end of the article
} 

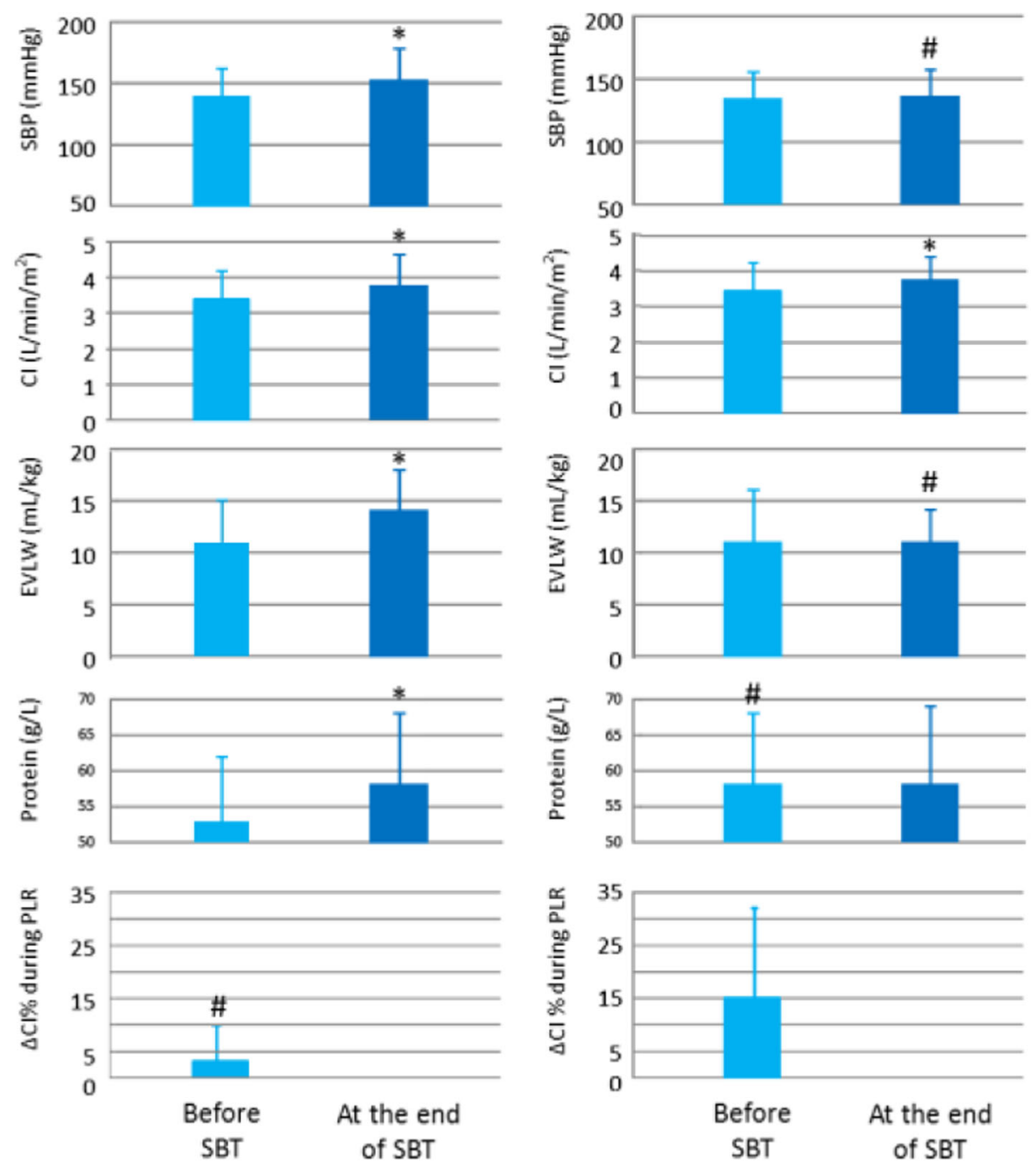

Cases with WiPO

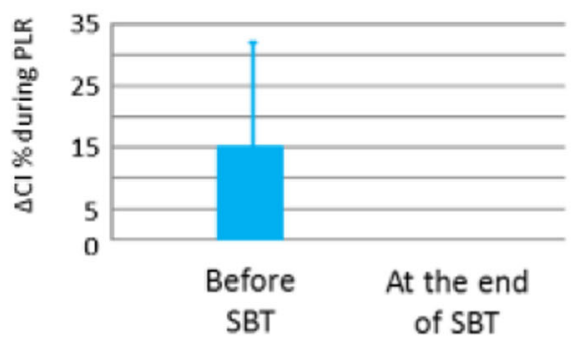

Cases without WiPO

Fig. 3 Changes in haemodynamic variables, plasma protein concentration, and extravascular lung water during the spontaneous breathing trial $(S B T) .{ }^{*} p<0.05$ at the end of SBT vs. before SBT; ${ }^{\#} p<0.05$ cases without WiPO vs. cases with WiPO. Cl cardiac index, EVLW extravascular lung water, $P L R$ passive leg raising, SBP systolic arterial blood pressure, WiPO weaning-induced pulmonary oedema 